\title{
Ultraviolett - die unsichtbare Seite der Blumen
}

\author{
Klaus Lunau
}

\begin{abstract}
Flowers are communication structures between flowering plants and their pollinators, whereby visual colour signals play an outstanding role. Since no pollinator has a colour vision system similar to that in humans, the understanding of flower colours relies on the analysis with the eyes of the beholder. Ultraviolet light is exceptional in this context because it is invisible for humans, but perceivable for most pollinators and represents a colour component in many flowers. UV can contribute to the evolution of communication channels between flower and pollinator. UV-patterns on flowers can help to protect pollen against UV-radiation and likewise guide pollinators to find a landing place and access to floral reward; at the same time floral guides help to manipulate the flower-visitors' movements in order to optimize pollen deposition on the pollinator and on the stigma of conspecific flowers. Floral colour patterns produced by gloss can indicate glossy nectar droplets or mimic nectar. Using UV-photography and false colour photography in bee view illustrate the meaning of ultraviolet colour components on flowers.
\end{abstract}

\section{Zusammenfassung}

Blumen sind Kommunikationsstrukturen zwischen Blütenpflanzen und ihren Bestäubern. Dabei spielen visuelle Farbsignale eine herausragende Rolle. Da kein Bestäuber über ein dem Menschen ähnliches Farbsehsystem verfügt, beruht das Verstehen von Blumenfarben auf einer Analyse mit den Augen des betrachtenden Blütenbesuchers. Ultraviolettes Licht hat dabei eine Sonderstellung, da es für Menschen unsichtbar, für die meisten Blütenbesucher aber wahrnehmbar und eine von vielen Blüten ausgebildete Farbkomponente bildet. UV kann zu Kommunikation zwischen Blüte und Bestäuber entscheidend beitragen. UVMuster auf Blüten schützen Pollen vor UV-Strahlung und sind gleichzeitig wegweisende Strukturen, mit deren Hilfe Blütenbesucher einen Landeplatz und eine Belohnung finden und gleichzeitig so manipuliert werden, dass die Übertragung von Pollen auf den Bestäuber oder von diesem auf die Narbe anderer Blüten wahrscheinlicher wird. Blütenfarbmuster, die durch Glanz erzeugt werden, können auf sichtbar glänzenden Nektar hinweisen oder diesen vortäuschen. Mit UV-Aufnahmen und Falschfarbenfotos in Bienensicht wird die Bedeutung ultravioletter Farbkomponenten auf Blüten deutlich gemacht.

\section{Einführung}

Im Jahre 1793 legte der Spandauer Gymnasialdirektor Christian Konrad Sprengel mit seinem Werk „Das entdeckte Geheimnis der Natur im Bau und in der Befruchtung der Blumen“ den Grundstock für eine moderne Blütenökologie (Sprengel 1793). Seine Beobachtung, dass Insekten als Blütenbestäuber fungieren, bildete die Grundlage dafür, Blumen als Locksignale für Blütenbesucher zu sehen. Der gedankliche Durchbruch findet sich am Anfang der Einleitung „Im folgenden Sommer untersuchte ich das Vergiß mein nicht (Myosotis palustris). ... Zugleich aber fiel mir der gelbe Ring auf, welcher die Öffnung der Kronenröhre umgiebt, und gegen die himmelblaue Farbe des Kronensaumes so schön absticht. ... Sollte die Natur wohl diesen Ring zu dem Ende besonders gefärbt haben, damit derselbe den Insekten den Weg zum Safthalter zeige. ... Nun schloß ich vom Theil auf das Ganze. Wenn, dachte ich, die Krone der Insekten wegen an einer besonderen Stelle besonders gefärbt ist, so ist sie überhaupt der Insekten wegen gefärbt..." Nun ist es heraus: Blumen sind nicht etwa zur Erbauung des Menschen geschaffen, sondern sind Schauapparate zur Anlockung für Insektenbestäuber.

Es ist erstaunlich, dass Sprengel diese Erkenntnisse ohne eine genaue Kenntnis des Farbensehens von Insekten erlangte. Durch UV-Fotografie können für den Menschen verborgene, für UV-tüchtige Insekten jedoch sichtbare Informationen visualisiert und so ein vertieftes Verständnis der Blumenfarben erlangt werden. Bei der UVFotografie wird mittels eines ausschließlich für UV-Licht durchlässigen Filters, eines UV-durchlässigen Objektivs und einer UV-empfindlichen Kamera fotografiert. Die Falschfarbenfotografie in Bienensicht geht noch einen Schritt weiter, indem sie mit Ultraviolett, Blau und Grün genau die Wellenlängenbereiche berücksichtigt, die Bienen und andere Insekten sehen können, jedoch das für viele Insekten unsichtbare Rot verwirft. Da Reflexion im roten Wellenlängenbereich in Farben wie Gelb, Purpur und Weiß enthalten ist und wir uns gedanklich nur schwer vorstellen können, wie ein 


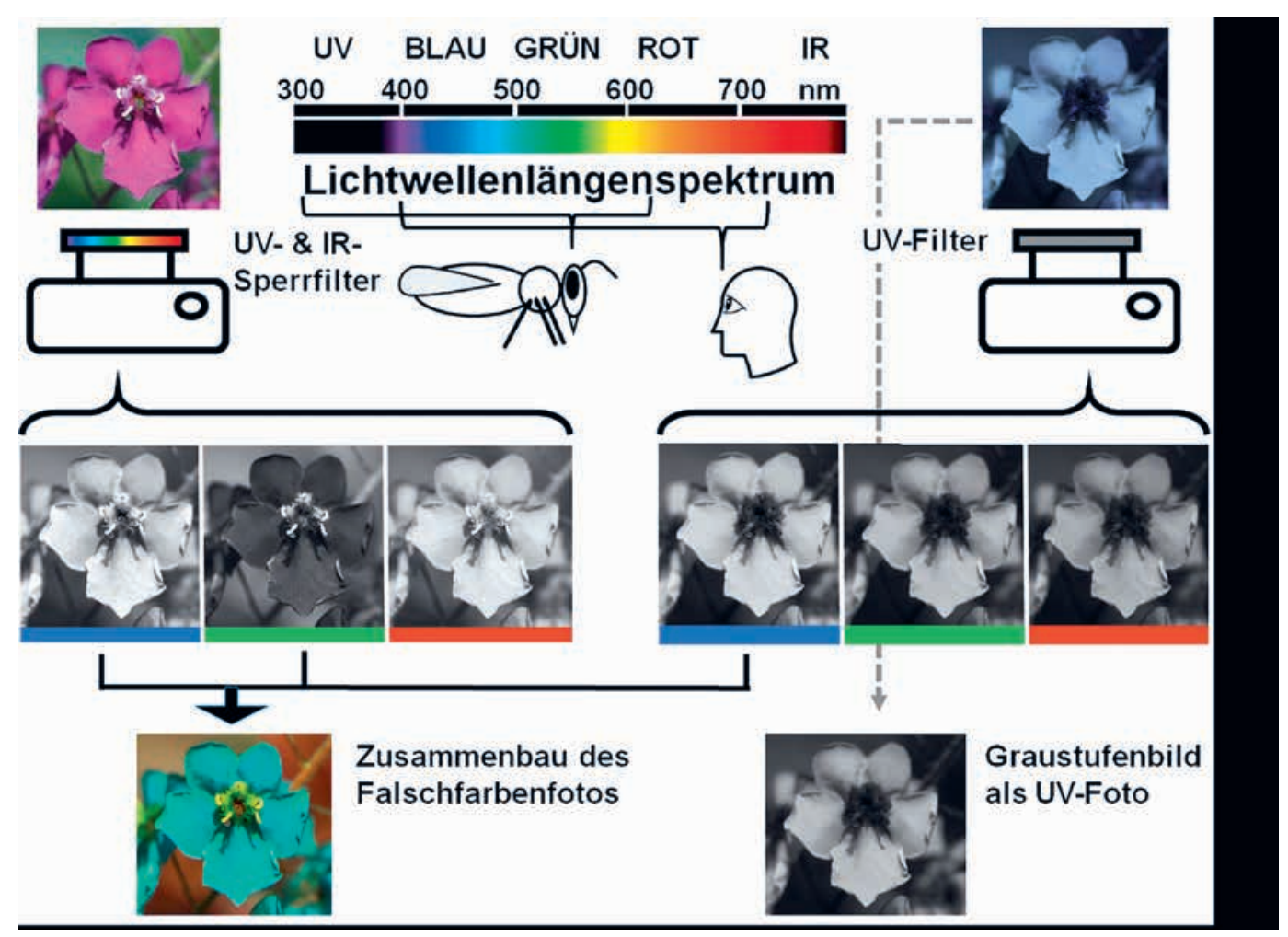

Abb. 1: Falschfarbenfotografie und UV-Fotografie einer Blüte von Verbascum phoeniceum L. im Vergleich. Oben: Ansicht im Display der Digitalkamera mit UV- und IR-Sperrfilter, bzw. UV-Filter. Mitte: Bildzerlegung in Blau-, Grün- und Rot-Kanal. Unten: Zusammenbau des Falschfarbenfotos aus dem Blau- und Grün-Kanal des Farbfotos und aus dem auch die UV-Information enthaltenden Blau-Kanal des UV-Fotos, bzw. Umwandlung in ein Graustufenbild als UV-Foto. (Grafik: K. LunaU)

UV-tüchtiges, aber Rot-unempfindliches Insekt diese Farben wahrnimmt, ob sie nun UV-reflektieren oder nicht, gibt die Falschfarbenfotografie in Bienensicht bessere Anschauungshilfe, wie Bienen Blumen sehen. Die Farbaufnahme und die UVAufnahme werden von der Kamera als Farbaufnahme abgespeichert und müssen zur Herstellung eines Falschfarbenfotos in ihre Komponenten zerlegt werden. Bei der UV-Aufnahme hängt es dabei vom Kameramodell ab, welcher Sensor der empfindlichste ist und das UV-Muster am besten wiedergibt; in dem in Abb. 1 dargestellten Fall ist es der Blau-Sensor. Bei der Falschfarbenfotografie in Bienensicht werden also eine UV-Aufnahme und eine Farbaufnahme in ihre Komponenten zerlegt und so zusammengebaut, so dass auf dem Falschfarbenfoto UV (Blau-Kanal der UV-Aufnahme) als Blau, Blau (Blau-Kanal der Farbaufnahme) als Grün und Grün (Grün-Kanal der Farb- aufnahme) als Rot dargestellt und Rot verworfen wird (Abb. 1; Lunau \& Verhoeven 2017). Im Unterschied zur Kombination von Farbfoto und UV-Foto enthält ein Falschfarbenfoto alle und ausschließlich nur die für Bienen sichtbaren Wellenlängenbereiche.

Die Falschfarben zeigen die Farbkontraste auf, jedoch nicht die Bienenfarben, denn Blau stellt ja Bienen-Ultraviolett dar, Grün entspricht BienenBlau und Rot entspricht Bienen-Grün. Schwarz ohne UV ist Bienen-Schwarz und Schwarz mit UV ist Bienen-Ultraviolett. Weiß mit UV ist Bienen-Weiß, während ein UV-absorbierendes Weiß Bienen-BlauGrün ist und im Falschfarbenbild gelb erscheint.

Die Falschfarbenfotografie ist eine Methode, die im Unterschied zur Reflexionsspektrophotometrie auch kleinste und ungewöhnlich geformte 


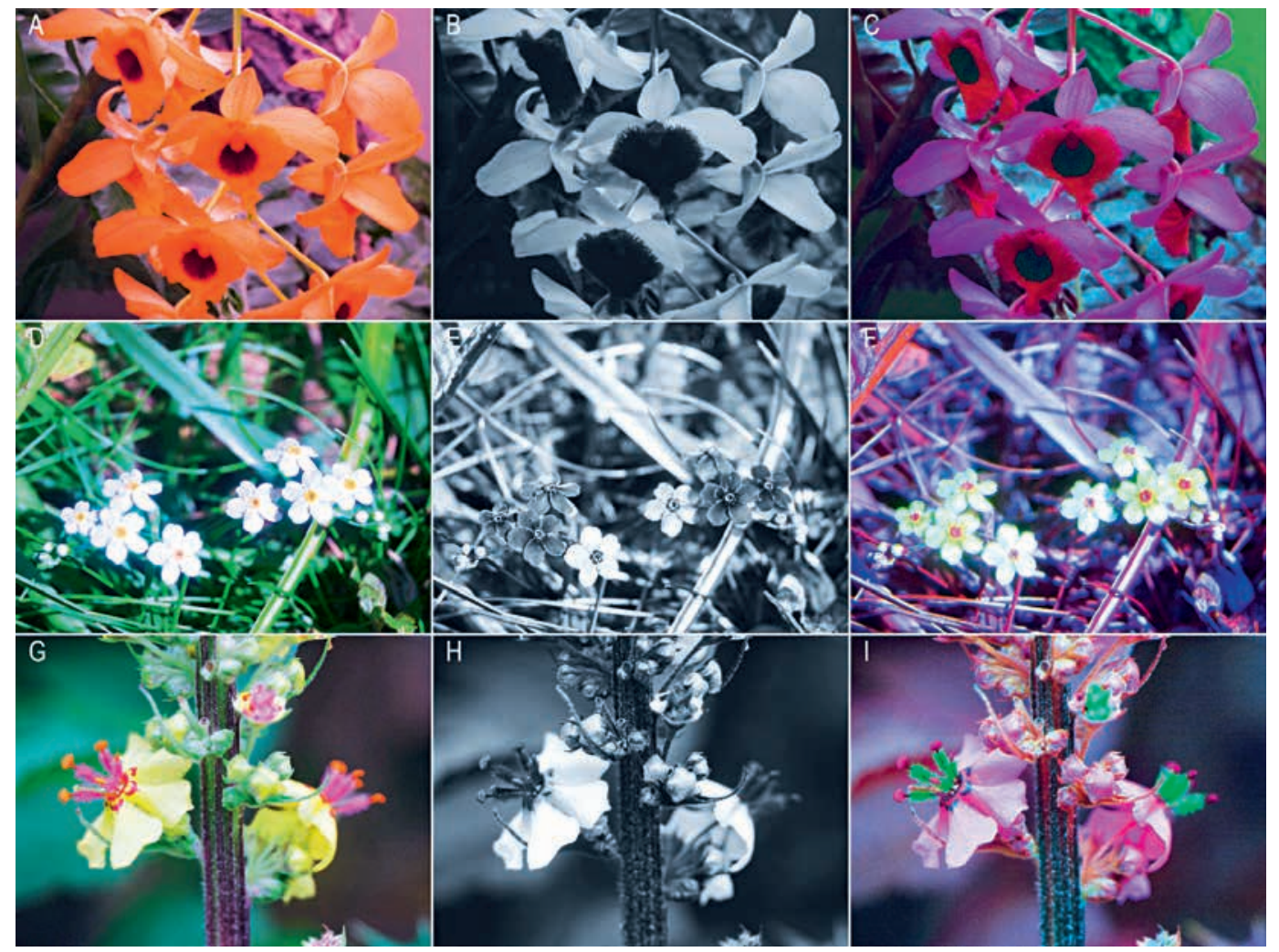

Abb. 2: Farb-, UV- und Falschfarbenaufnahme von A-C einer Blüte von Dendrobium fimbriatum LiNDL; D-F eines Blütenstandes vom Sumpfvergissmeinnicht Myosotis palustris L.; G-I einigen Blüten der Schwarzen Königskerze Verbascum nigrum L.. (Fotos: K. LunAu)

Strukturen erfassen kann wie die Filamenthaare der Schwarzen Königskerze (Abb. 2). Falschfarbenfotografie kann sogar als feldgängige Methode für lebende, seltene und geschützte Organismen eingesetzt werden und bei Nutzung einer UVTaschenlampe auch in geschlossenen Räumen und Gewächshäusern verwendet werden (Abb. 2).

\section{Kommunikation zwischen Blumen und Bestäubern}

Durch die experimentelle Blütenbiologie entwickelte sich die Erkenntnis, dass das Farbensehen von Bestäubern sich von dem des Menschen unterscheidet. Bahnbrechend sind die Versuche von KARL vON Frisch (1915) zum Farbensehen von Honigbienen, in denen er zeigt, dass auf einen Farbton dressierte Honigbienen diese Farbe von 15 verschiedenen Graustufen unterscheiden können; wären Bienen nicht farbtüchtig, so sollten die Bienen die Farbe mit der ihnen gleichhell er- scheinenden Graustufe verwechseln. Die Bienen unterscheiden aber klar Farben von allen Graustufen. Karl von Frisch schloss, dass Bienen die Fähigkeit des Farbensehens besitzen, da sie die Farbe allein auf Grund ihrer Farbigkeit und unabhängig von ihrer Helligkeit unterscheiden konnten. Nur bei tiefroten Farbkarten funktioniert die Unterscheidung nicht, da Honigbienen für rotes Licht wenig empfindlich sind. Sein Schüler KarL DAumer (1958) wusste bereits, dass Bienen zwar kein Rot, aber neben Grün und Blau auch UV sehen konnten und machte UV-Aufnahmen von Blüten, um diese für Menschen nicht sichtbare Dimension von Blüten zu erschließen. Inzwischen ist durch vergleichende Studien bekannt, dass sich das Farbensehen des Menschen vom Farbensehen aller Blütenbestäuber unterscheidet (LunAU \& Maier 1995; Lunau 2021). Während Bienen wie Menschen drei Farbrezeptoren besitzen, also trichromatisch sind, sind Kolibris und andere blü- 

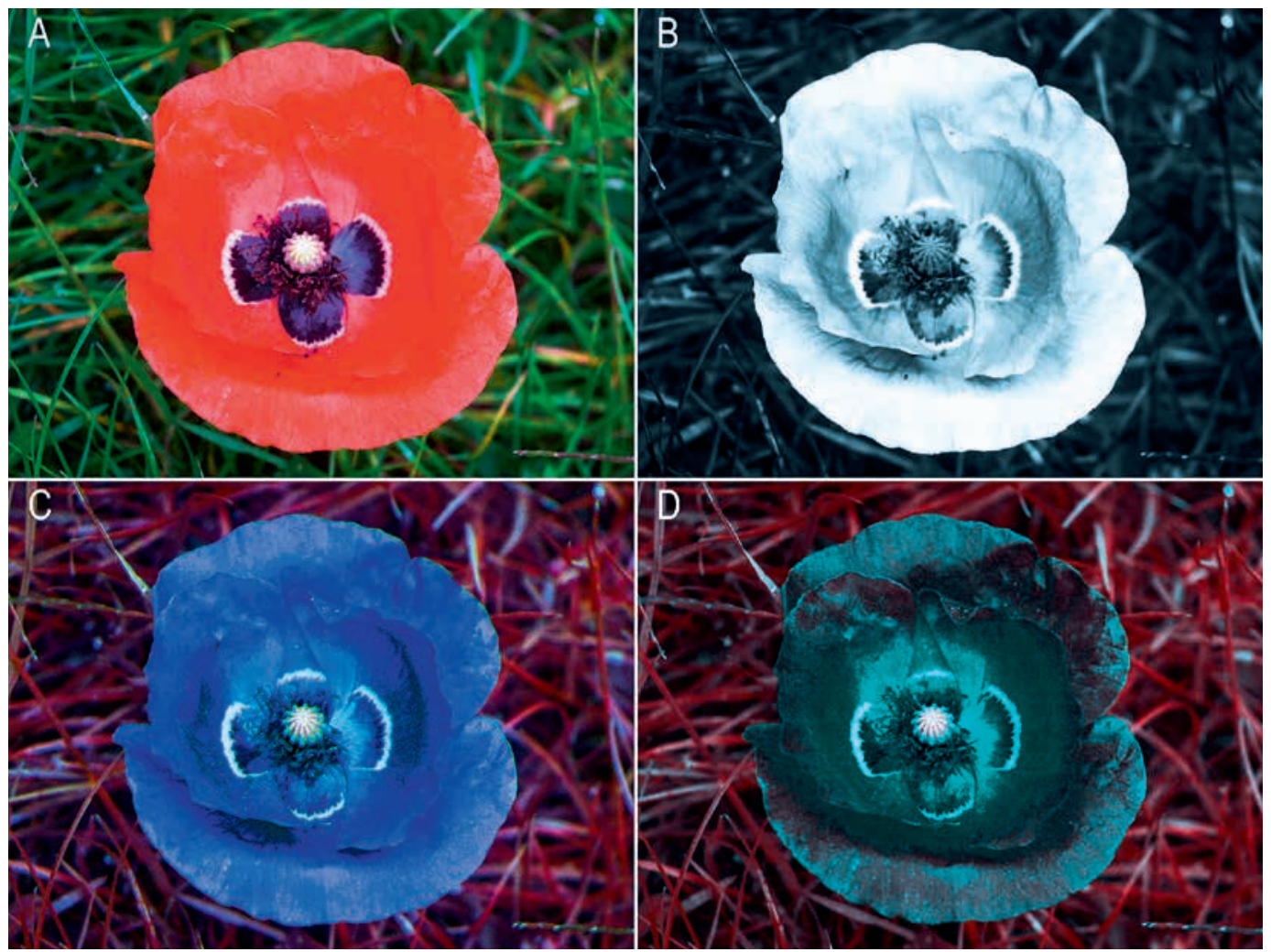

Abb. 3: A Farbfoto einer Klatschmohnblüte; B UV-Foto derselben Blüte; C Falschfarbenfoto in Bienensicht derselben Blüte; D Falschfarbenfoto in Bienensicht mit angenommener UV-Absorption derselben Blüte. (Fotos: K. LUNAU)

tenbesuchende Vögel tetrachromatisch mit Farbrezeptoren für ultraviolettes, blaues, grünes und rotes Licht; bei Nektarvögeln ist der UV-Rezeptor durch einen Violett-Rezeptor ersetzt (ÖDEEN \& HÅstad 2010). Blütenbestäubende Mäuse und Elefantenspringrüssler besitzen ein einfaches Farbensehen basierend auf zwei Farbrezeptortypen (Thüs et al. 2020). Fledermäuse können sich visuell auch ohne Farbrezeptoren mit den hoch lichtempfindlichen Stäbchen orientieren (WINTER et al. 2003). Schmetterlinge unterscheiden sich in ihrem Farbensehen und können mehr als 5 verschiedene Typen von Farbrezeptoren aufweisen (Arikawa 2003). Das Farbensehen von Fliegen beruht auf vier Rezeptortypen, darunter zwei maximal in UV empfindlichen Rezeptoren, und ist noch nicht völlig verstanden (AN et al. 2018).

Ein weiterer Unterschied zwischen dem Farbensehen von Mensch und Bienen liegt in der unterschiedlichen Verarbeitung von Farbreizen. Bienen beispielsweise reagieren kaum auf die Helligkeit eines Farbreizes. Erscheint Bienen jedoch ein Objekt unter kleinem Sehwinkel, weil es klein oder weit entfernt ist, orientieren sich Bienen ausschließlich nach der vom Grünrezeptor wahrgenommenen Helligkeit. Erst bei Annäherung an dieses Objekt, wenn der Sehwinkel einen kritischen Wert übersteigt, erkennen Bienen seine Farbe. Das hat Konsequenzen für die Blumenfarben, denn diese müssen, um für eine Biene erkennbar und attraktiv zu sein, sowohl einen Grünkontrast als auch einen Farbkontrast zur Umgebung bieten.

Die Unterschiede im wahrgenommenen Lichtspektrum zwischen Blütenbesuchern ermöglichen Blütenpflanzen eine Interaktion mit einem bestimmten Bestäuber in einem Wellenlängenbereich, in dem die Bestäuber, nicht aber 


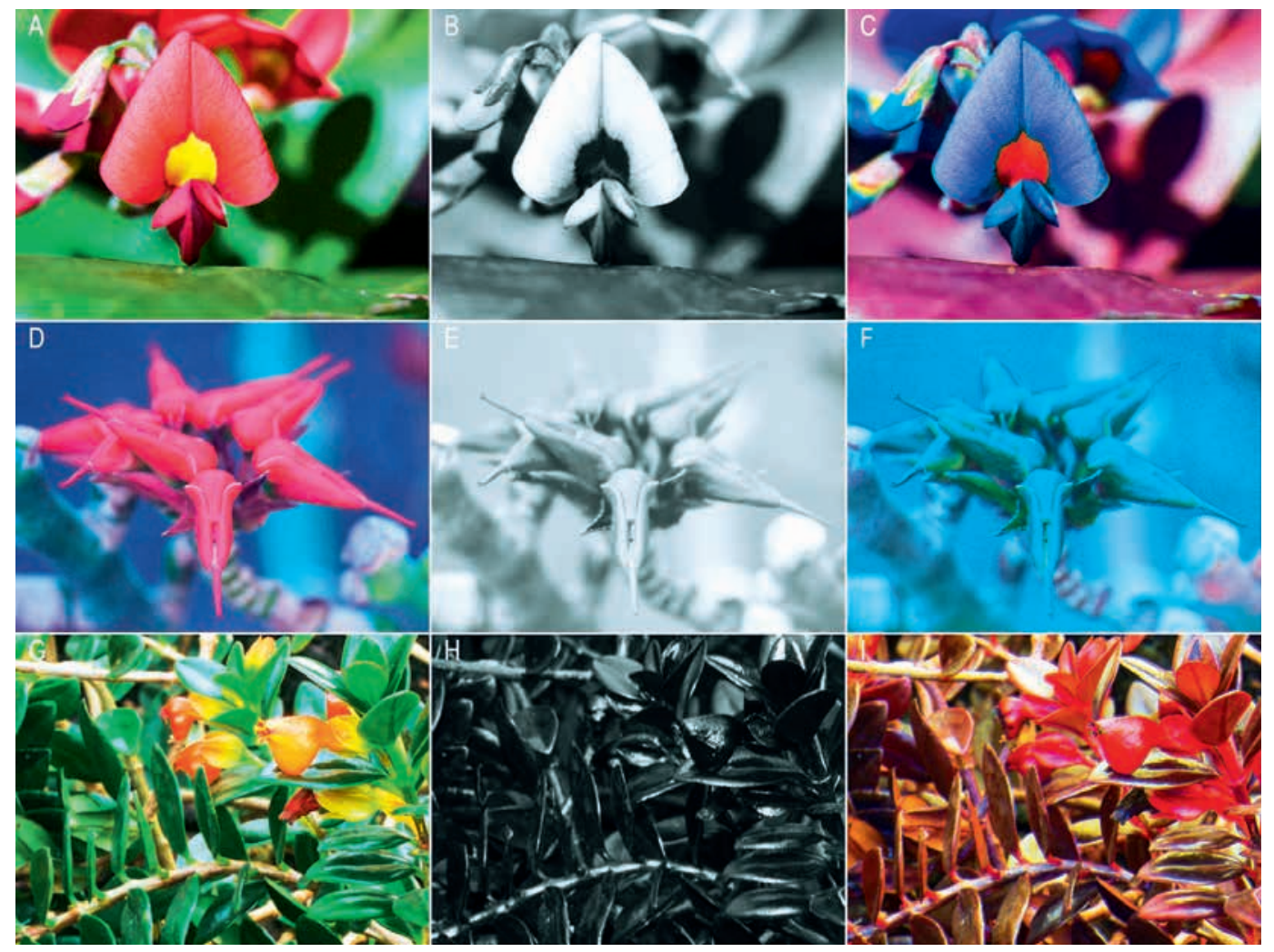

Abb. 4: Farb-, UV- und Falschfarbenaufnahme von roten Bienen- und Vogelblumen. A-C von Bienen bestäubte Blüte von Kennedia lateritia; D-F von Vögeln bestäubter Blütenstand von Euphorbia tithymaloides; G-I von Vögeln bestäubte Blüte von Nemananthus strigillosus. (Fotos: K. LunAu)

Nektardiebe oder Pollenräuber empfindlich sind. Ein Beispiel bildet der Klatschmohn Papaver rhoeas; seine roten Blüten locken Käfer an, die rotes Licht sehen können und sogar präferieren. Sie nutzen die Blüten auch zur Partnerfindung. In Mitteleuropa kommen diese Käfer nicht vor. Der Klatschmohn weist hier eine starke Reflexion im ultravioletten Wellenlängenbereich auf, wodurch er den rotblinden, aber UV-tüchtigen Bienen in Bienen-Ultraviolett erscheint, während der ostmediterrane Klatschmohn ohne UVReflexion den Bienen wenig attraktiv in BienenSchwarz erscheint (Abb. 3; Martínez-Harms et al. 2020).

Bienen präferieren keinen bestimmten Farbton, sondern alle Farben, die ihnen subjektiv gesättigt erscheinen. Weiß, Schwarz und Grau sind ungesättigte Farben, reine Farben ohne
Beimengung von Weiß sind dagegen gesättigte, umgangssprachlich knallige Farben. Diese Farbpräferenz von Bienen führt dazu, dass Blüten den Besuch von Bienen dadurch einschränken können, indem sie bienensubjektiv ungesättigte Farben aufweisen. Dieser Fall liegt bei den Vogelblumen vor (LuNAu et al. 2011). Vogelbestäubte Blüten sind weltweit ganz überwiegend rot gefärbt (CHEN et al. 2020). Auch in diesem Fall vermutete man zunächst eine Präferenz für rote Farben bei Vögeln; jedoch scheiterten alle Versuche mit unvoreingenommenen blütenbesuchenden Vögeln, eine angeborene Farbpräferenz nachzuweisen. Es gibt jedoch Unterschiede zwischen roten Vogelblumen und roten Bienenblumen (Lunau et al. 2011). Vogelbestäubte rote Blüten absorbieren meist ultraviolettes Licht, während bienenbestäubte rote Blüten es meist reflektieren (Abb. 4). Spontanwahltests mit Prachtbienen und Kolibris ergaben, 

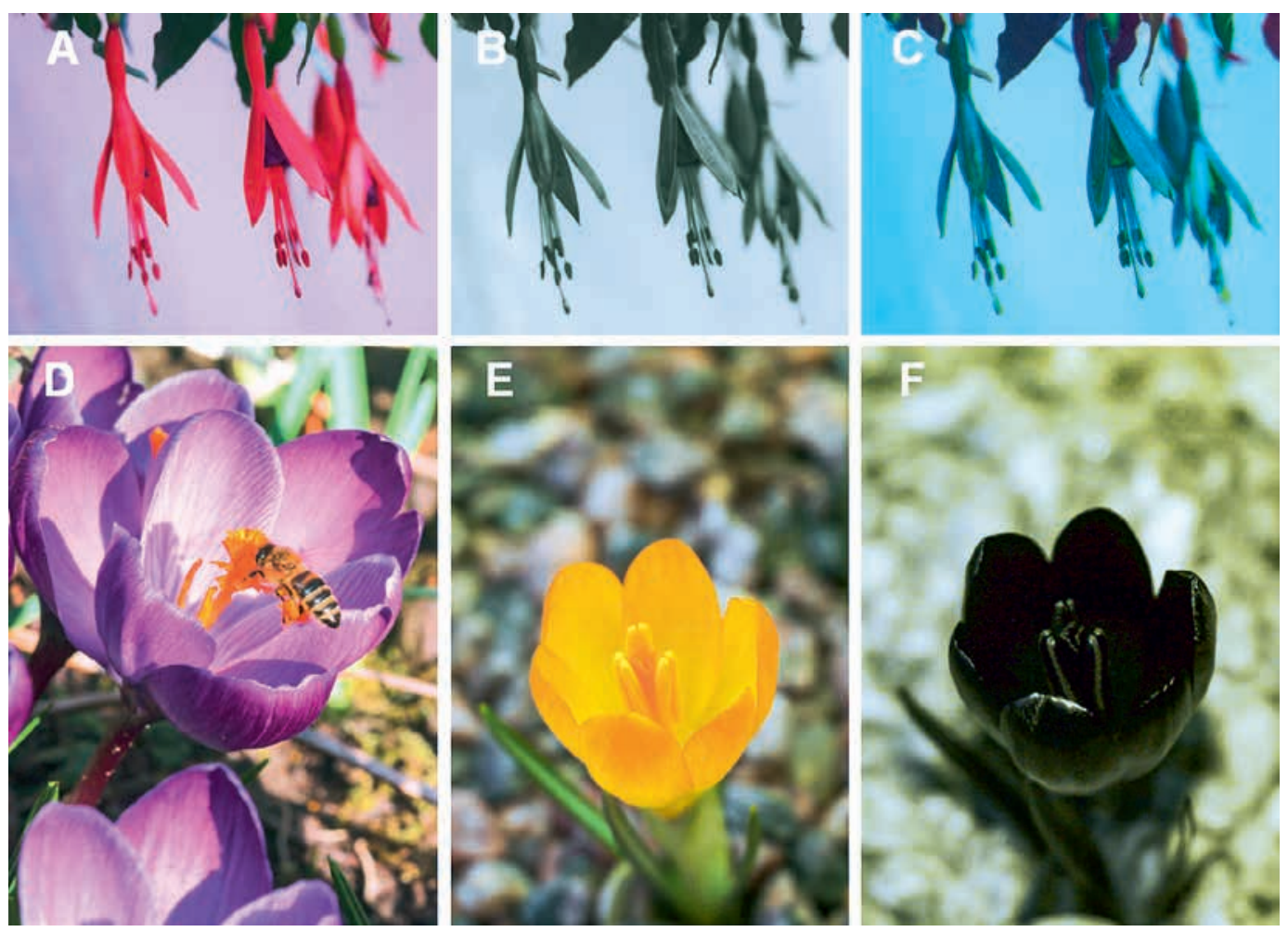

Abb. 5: A-C Farb-, UV- und Falschfarbenaufnahme von Blüten einer Fuchsie. Fuchsien reflektieren gleichmäßig etwas UVLicht. In unseren Gärten wird sie häufig von Bienen aufgesucht. In ihrer südamerikanischen Heimat sind Kolibris wichtige Bestäuber. D Honigbiene im Landeanflug auf die orangefarbenen Narbenäste eines blauen Gartenkrokus. B Farbaufnahme eines gelbblühenden Crocus cvijicii. C UV-Aufnahme eines gelbblühenden C. cvijicii. (Fotos: K. LunAu)

dass Kolibris rote Farben mit und ohne UV-Reflexion gleich häufig wählen, neotropische Prachtbienen aber Rot mit UV signifikant gegenüber Rot ohne UV bevorzugen. Die zweithäufigste Farbe von vogelbestäubten Blüten ist Weiß. Weiße bienenbestäubte Blüten absorbieren meist UV, während weiße vogelbestäubte Blüten UV häufig reflektieren. Im Spontanwahltest ziehen die Prachtbienen Weiß ohne UV einem Weiß mit UV vor, jedoch nicht so die Kolibris. Offenbar reduzieren vogelbestäubte Blüten den Besuch von Nektar raubenden und Pollen stehlenden Bienen, indem sie Farben ausbilden, die für Bienen wenig gesättigt und daher wenig attraktiv, für Vögel aber auffällig und anlockend sind. Offen ist weiterhin die Frage, warum viele, aber nicht alle Vogelblumen Blütenbesuch von Bienen durch eine für Bienen unattraktive Farbe reduzieren. Man darf annehmen, dass die hohe Nektarmenge vogelbestäubter Blüten diese auch ohne visuelle Auf- fälligkeit für Bienen attraktiv macht (Abb. 5). Es gibt Hinweise, dass Blüten, die bereits durch ihre Morphologie oder durch die glatte Oberfläche der Blütenkrone, die Bienen keinen Halt bietet, vor Bienen als Nektarräuber geschützt sind, auf einen sensorischen Ausschluss über die Blütenfarbe verzichten (PAPIOREK et al. 2014).

Für gelbe Vogelblumen ist ein anderer Mechanismus des sensorischen Ausschlusses von Bienen belegt. Gelbe Vogelblumen absorbieren komplett UV und weisen kein Farbmuster auf, während gelbe Bienenblumen UV-Licht reflektieren und oftmals ein UV-absorbierendes Zentrum besitzen (PAPIOREK et al. 2016). Man vermutet, dass Bienen das UV-Muster zum schnellen Auffinden der Blütenbelohnung benötigen und bei gelben Blüten ohne UV-Muster Probleme oder Zeitverlust beim Aufsuchen der Nahrung in den Blüten haben (Abb. 4). 


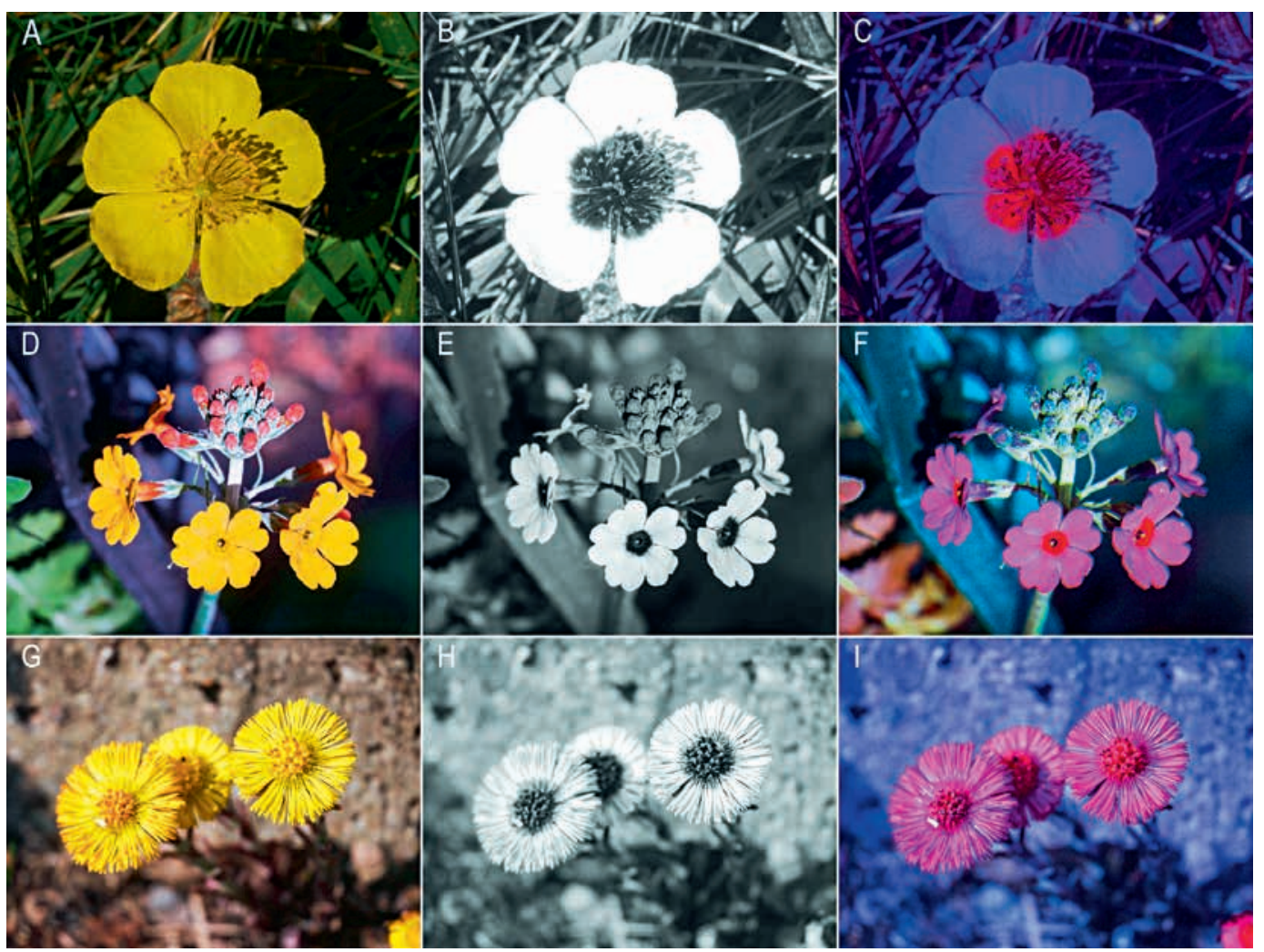

Abb. 6: Farb-, UV- und Falschfarbenaufnahme von UV-Mustern. A-C Gelbes Sonnenröschen Helianthemum nummularium; D-F Etagen-Schlüsselblume Primula bulleyana; G-I Huflattich Tussilago farfara. (Fotos: K. LunAu)

Die Bedeutung von Farbmustern wird deutlich bei Krokussen. Blaublühende Krokusse locken bestäubende Bienen und Schwebfliegen beim Blütenbesuch auf die orangefarbene Narbe, wodurch zunächst anhaftender Pollen zur Fremdbestäubung gelangt. Gelbblühende Krokusse wie Crocus cvijicii absorbieren aus unbekannten Gründen UV-Licht, so dass die Blüten kein Farbmuster aufweisen und auf die farblich kontrastierende Narbe als Landeplattform für Bestäuber verzichten (Abb. 5). Das Phänomen ist in der gesamten Gattung Crocus verbreitet (LunAu et al. 2016).

\section{Blütenfarbmuster aus Sicht der Bienen}

Saftmale nannte SPRENGEL jene Blütenstrukturen, an denen sich Blütenbesucher an oder auf der Blüte orientieren können. Der englische Fachbegriff „honeyguides“ hält sich hartnäckig, obwohl Blüten keinen Honig, sondern Nektar anbieten und viele Blütenmale Pollen- und Antherenimitationen dar- stellen und die gelbe, UV-absorbierende Farbe von Pollen und Antheren imitieren (Abb. 5; Osche 1979). Diese Pollen- und Antherenmimikry führt nur in einigen Fällen zu Täuschung von Blütenbesuchern. Gelbe, UV-absorbierende Blütenmale können verschiedene Blütentypen (z. B. männliche oder weibliche) oder Blühphasen für einen Blütenbesucher schwerer unterscheidbar machen. Bei Kürbisgewächsen und Begonien ähneln sich staminate (männliche) und pistillate (weibliche) Blüten dadurch, dass Form und Farbe der Narbe dem Androeceum gleicht (Abb. 6). Bei nektarlosen Begonien enthalten die pistillaten Blüten weder Pollen noch Nektar und alle Blütenbesucher werden getäuscht. Die Blütenmorphen der heterostylen Köcherblümchen unterscheiden sich in der Länge der Staubgefäße und Griffel. Das wäre für manche Bienen sicher ein Grund, die Morphe mit der zu ihrer Körpergröße passenden Staubgefäßlänge zu bevorzugen; die Unterscheidung 


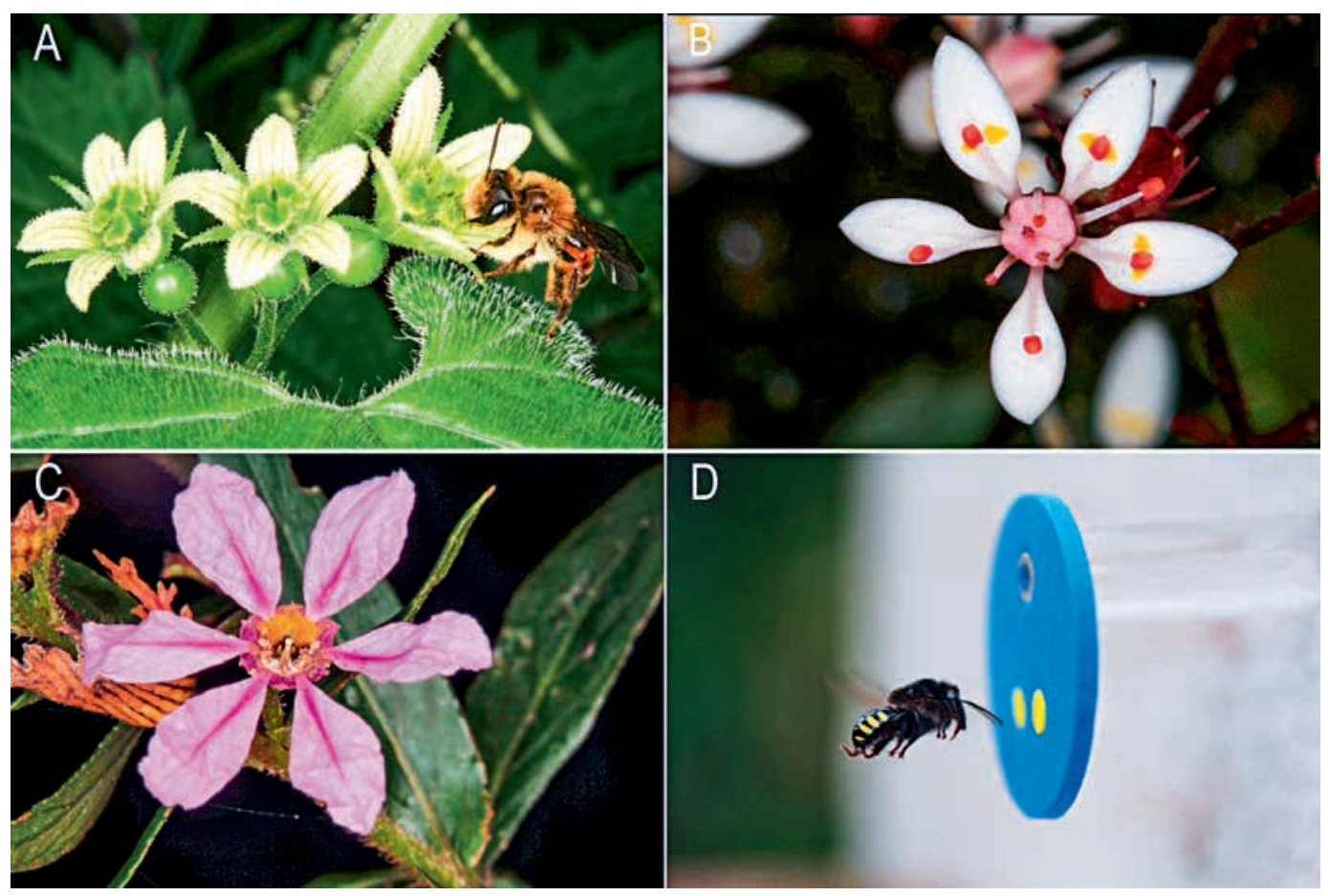

Abb. 7: A Pistillate Blüte der zweihäusigen Roten Zaunrübe Bryonia dioica mit der Sandbiene Andrena florea; B Männliche Blühphase einer Blüte des Stern-Steinbrechs Saxifraga stellaris; C Blütenmorphe des heterostylen Köcherblümchens Cuphea calophylla cf; D Anflug einer Arbeiterin der Stachellosen Biene Melipona quadrifasciata an eine Blütenattrappe mit gelbem UVabsorbierenden Blütenmal. (Fotos: K. LunAu)

der Morphen wird den Bienen jedoch dadurch erschwert, dass alle drei Morphen ein auffälliges gelbes, UV-absorbierendes Blütenmal an identischer Position aufweisen, während die Staubgefäße selbst unauffällig und verborgen sind (Abb. 7). Dichogame Blüten besitzen eine staminate Blühphase mit besserem Pollenangebot als die pistillate Blühphase; gelbe UV-absorbierende Pollen imitierende Blütenmale in Kombination mit wenig attraktiven Staubgefäßen wie beim Stern-Steinbrech sorgen für eine erschwerte Unterscheidung der Blühphasen (PoHL et al. 2008). Viele Blüten nutzen die Pollen und Antheren imitierenden Blütenmale, um Bienen und Schwebfliegen den Zugang zur Blütenbelohnung anzuzeigen (Abb. 6; LunAu et al. 2006, An et al. 2018). Diese fliegen Blütenmale angeborenermaßen zielgerichtet an, bzw. betupfen sie mit dem Rüssel. Für Menschen kaum oder gar nicht sichtbare gelbe, UV-absorbierende Blütenmale auf meist gelben, UV-reflektierenden Blüten haben sogar einen eigenen Begriff erhalten, UV-
Bull's eye, nach der kleinen Mitte auf der Dartscheibe. Beispiele für die konvergente Bildung von UV-Mustern sind das Gelbe Sonnenröschen mit UV-absorbierenden Blütenblattbasen und Staubgefäßen, die Etagen-Schlüsselblume mit UV-absorbierendem Kronenzentrum und der Huflattich Tussilago farfara mit UV-absorbierenden Scheibenblüten (Abb. 6).

UV-absorbierende Blütenmale haben noch eine weitere Funktion. Sie verhindern, dass reflektiertes UV-Licht die Staubgefäße oder die Narbe erreicht wie beim Sonnenröschen (Abb. 6). Die Größe von Blütenmalen wird daher auch von der Intensität der UV-Strahlung in der Umgebung beeinflusst (Koski \& Ashman 2016).

Wenn Blüten sich umfärben, sind es oftmals lediglich die Blütenmale, die ihre Farbe ändern. Dann wechseln meist gelbe und UV-absorbierende Blütenmale in eine rote oder weiße Farbe und 

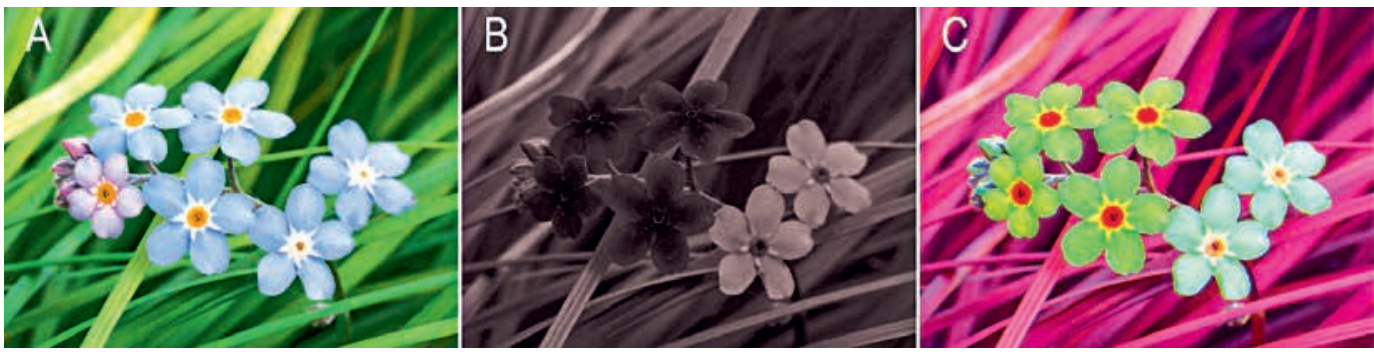

Abb. 8: Farb-, UV- und Falschfarbenaufnahme von UV-Mustern bei einer Gartenform des Vergissmeinnicht. (Fotos: K. LunAU)

gleichzeitig stellt die Blüte die Nektarproduktion ein, welkt aber nicht (Abb. 8). Dadurch gelingt es den Pflanzen, mittels der großen Fläche aus vielen Blüten Bestäuber über eine größere Entfernung anzulocken und ihnen in der Nähe nektarproduzierende Blüten ehrlich anzuzeigen. Die Nektar führenden Blüten erscheinen auch in den für Bienen und Schwebfliegen attraktiven Farben (LunAu 1996).

\section{Ungewöhnliche Blütenfarben durch Glanz} Die meisten Blumenfarben werden durch Pigmente wie Carotinoide, Betalaine, Anthocyane und andere Flavonoide erzeugt. Es mehren sich jedoch Hinweise auf die Bedeutung von physikalischen Farben an Blumen, etwa Fluoreszenzfarben, Polarisationsmuster, Schillerfarben, blauer Halo und Glanz (Moyroud \& Glover 2016; Moyroud et al. 2017). Glanz beruht auf einer Totalreflexion
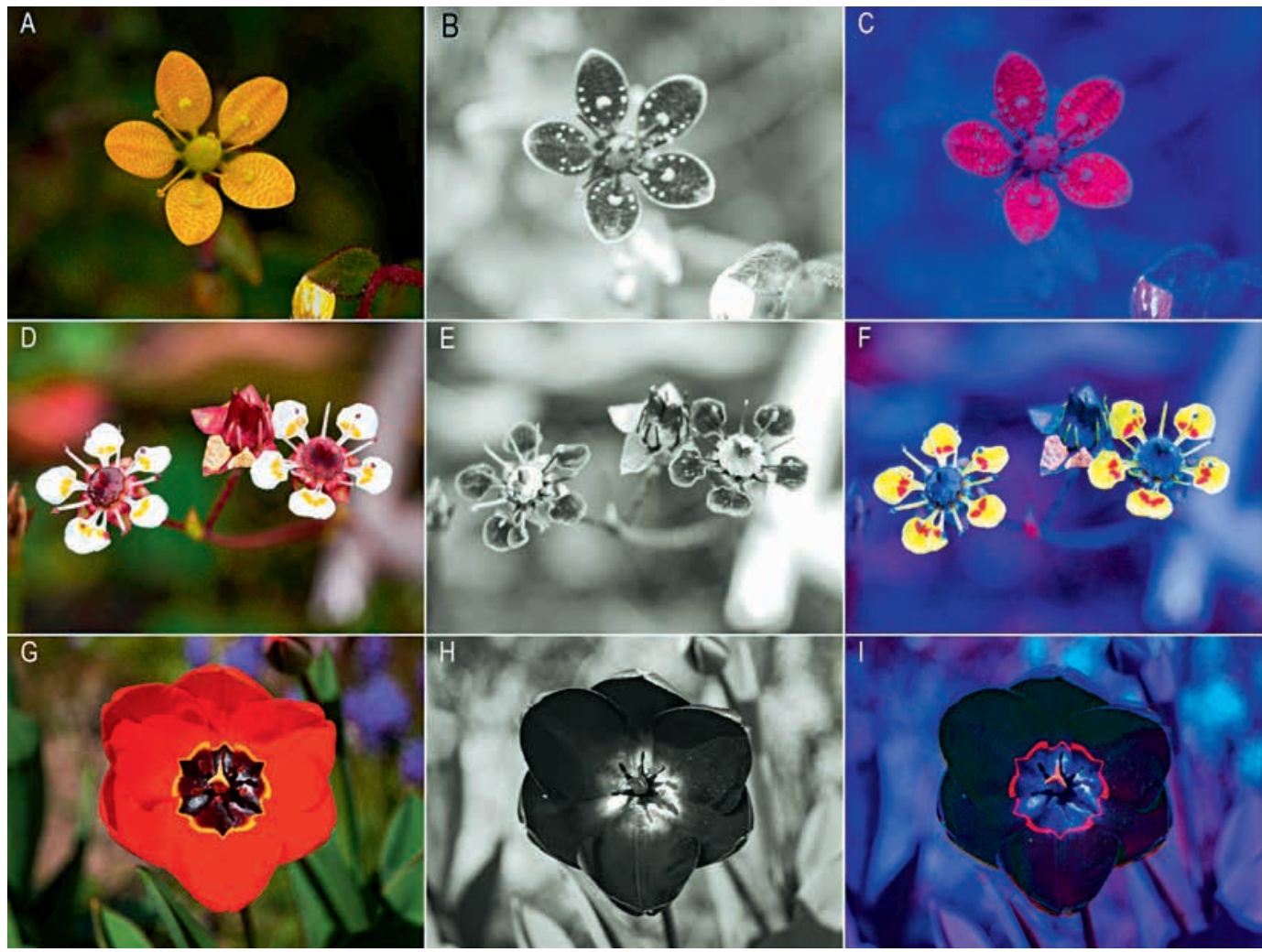

Abb. 9: Farb-, UV- und Falschfarbenaufnahme von Blumen mit Glanzmustern. A-C Saxifraga nigroglandulosa; D-F Saxifraga melanocentra; G-I Gartentulpe Tulipa gesneriana. (Fotos: K. LunAu) 


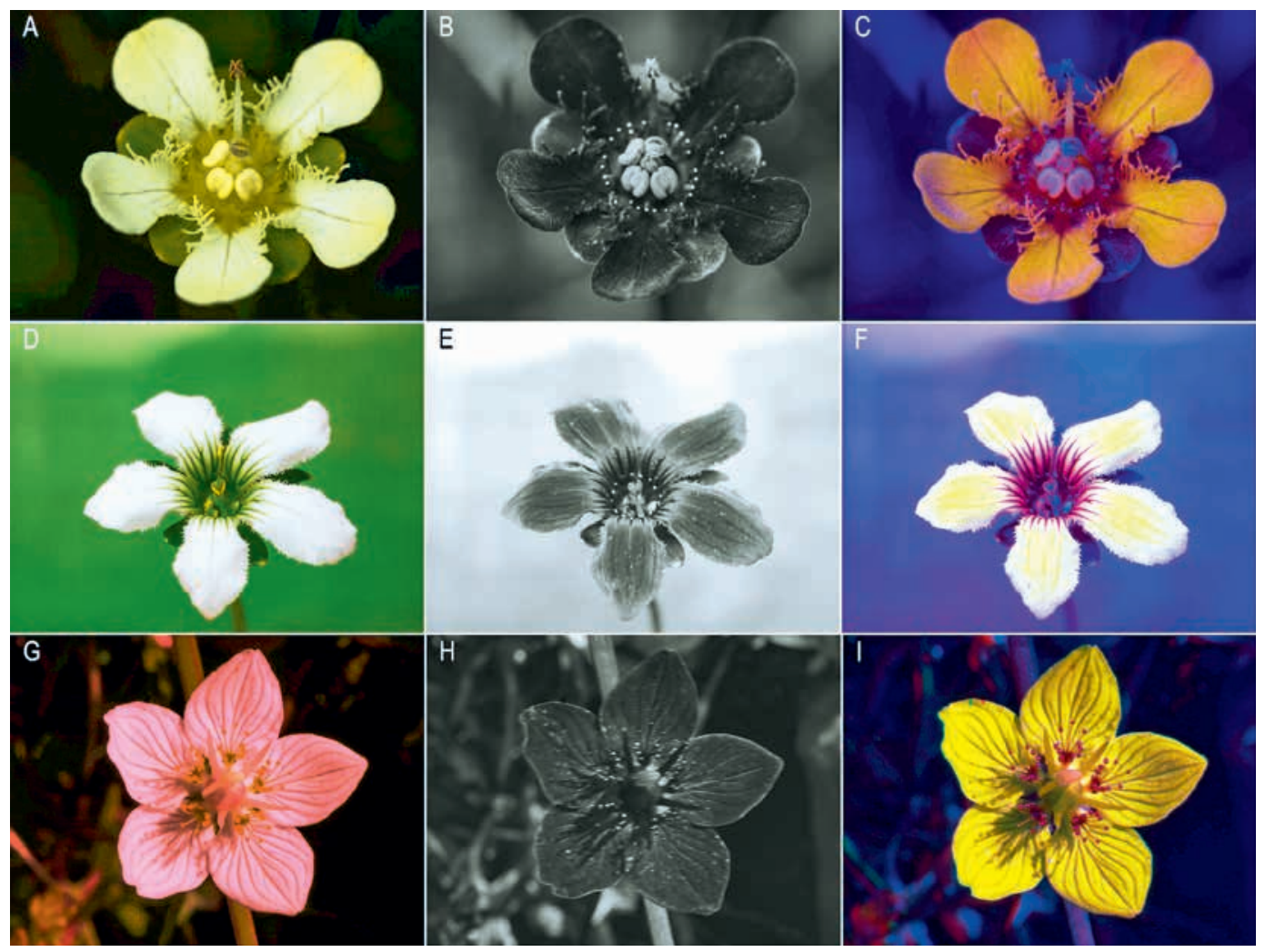

Abb. 10: Farb-, UV- und Falschfarbenaufnahme von Parnassien mit Glanzmustern auf den Staminodien. A-C Parnassia wightiana; D-F Parnassia delavayi; G-I Sumpfherzblatt Parnassia palustris. (Fotos: K. LunAu)

von Licht; eine glänzende Stelle auf der Blüte besitzt also dasselbe Lichtspektrum wie die Lichtquelle, die meist die Sonne ist. Glanzeffekte sind winkelabhängig, der Einstrahlungswinkel gleicht dem Glanzwinkel des reflektierten Lichtes. Blüten haben meist keine glatten Oberflächen, an denen Glanz entsteht, denn die epidermalen Zellen sind oft konisch. Diese konische Zellform beeinflusst die Haltemöglichkeiten für Insekten, die Benetzbarkeit und auch die Blütenfarben, indem sie wie Linsen das einfallende Licht auf die pigmentführende Schicht fokussiert. Genau genommen besitzen auch konische Zellen Glanz, der jedoch auf einen winzig kleinen Bereich reduziert ist, während glatte Zellen großflächig glänzen. Glanz ist daher in der Lage, dem Betrachter Informationen über die Form eines Objektes zu geben. Freiliegende Nektartropfen sind für Blütenbesucher an ihrem Glanz zu erkennen. Eine Studie zeigt, dass glänzende Protuberanzen Nektartropfen imitieren können (LuNAu et al. 2020). Wiederum trägt die
UV-Komponente zur besseren Erkennbarkeit des Glanzes bei. Auf dunklen und UV-absorbierenden Flächen kontrastieren glänzende Stellen einfach stärker. Offenbar nutzen viele Blüten dieses Phänomen, um den von ihnen dargebotenen Nektar besser zur Geltung zu bringen, um den Zugang zum Nektar anzuzeigen oder um Nektar vorzutäuschen. Auf den Blütenblättern von Saxifraga nigroglandulosa sind jeweils zwei Reihen von kleinen, glänzenden Erhebungen zu sehen. Bei Saxifraga melanocentra glänzt Nektar auf dem dunklen Diskus. Die schwarzen Basen der Tepalen einer Gartentulpe sind ebenfalls im UV besonders gut zu erkennen. In allen Fällen zeigen die Falschfarbenbilder in Bienensicht die gute Erkennbarkeit für Bienen (Abb. 8).

In Blüten der Gattung Parnassia sind glänzende Staminodien ausgebildet (Abb. 10), die für den Menschen nicht immer, wohl aber für Bienen gut sichtbar sind. 


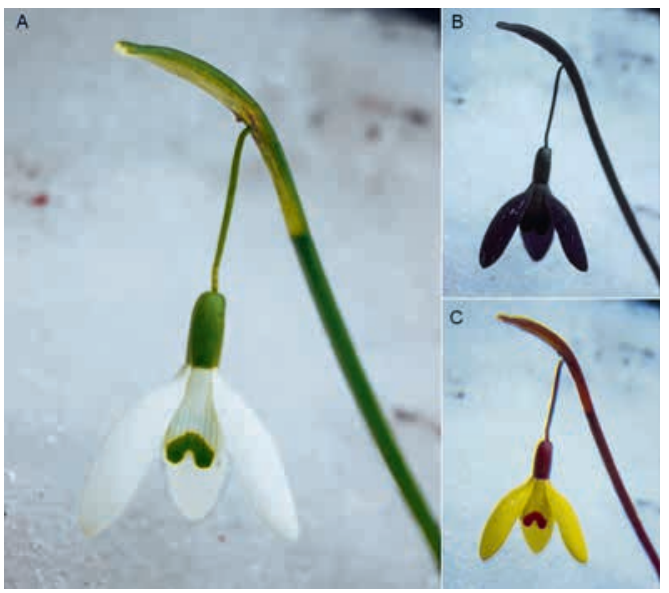

Abb. 11: A-C Farb-, UV- und Falschfarbenaufnahme eines Schneeglöckchens im Schnee. (Fotos: K. LunAU)

Abschließend sei noch einmal darauf hingewiesen, dass die Falschfarbenfotografie in Bienensicht zum besseren Verständnis der Blütenfarben, wie Bienen sie sehen, beitragen kann. Warum sollte ein im Schnee blühendes Schneeglöckchen ausgerechnet weiß sein (Abb. 11)? Die Falschfarbenaufnahme zeigt die Auffälligkeit eines Schneeglöckchens für eine anfliegende Biene.

\section{Literatur}

An, L., Neimann, A., Eberling, E., Algora, H., Brings, S. \& Lunau, K. 2018: The yellow specialist: Dronefly Eristalis tenax prefers different yellow colours for landing and proboscis extension. - J. Exp. Biol. 221: jeb184788.

ArikaWA, K. 2003: Spectral organization of the eye of a butterfly, Papilio. - J. Comp. Physiol. A 189: 791-800.

Chen, Z., Niu, Y., Liu, C.-Q. \& Sun, H. 2020: Red flowers differ in shades between pollination systems and across continents. - Ann. Bot. 126: 126: 837-848.

Daumer, K. 1958: Blumenfarben, wie sie die Bienen sehen. Z. vgl. Physiol. 41: 49-110.

Frisch, K. v.1914. Der Farbensinn und Formensinn der Bienen. - Zool. Jb., Abt. allg Zool. u. Physiol. 35: 1-188.

Koski, M.H. \& Ashman, T.-L. 2016: Macroevolutionary patterns of ultraviolet floral pigmentation explained by geography and associated bioclimatic factors. - New Phytol. 211: $708-718$.

LunAu, K. 1996: Unidirectionality of floral colour changes. Plant Syst. Evol. 200: 125-140.

Lunau, K. 2021: Mit allen Sinne - Wie Insekten ihre Welt erleben. - Entomologie heute 32: 11- 47.
Lunau, K., Fieselmann, G., Heuschen, B. \& van de Loo, A. 2006: Visual targeting of components of floral colour patterns in flower-naive bumblebees (Bombus terrestris; Apidae). - Naturwissenschaften 93: 325-328.

Lunau, K, Konzmann, S., Bossems, J. \& Harpke, D. 2016: A matter of contrast: Yellow flower colour constrains style length in Crocus species. - PLoS ONE 11; e0154728.

Lunau, K., Papiorek, S., Eltz, T. \& Sazima, M. 2011: Avoidance of achromatic colours by bees provides a private niche for hummingbirds. - J. Exp. Biol. 214: 1607-1612.

Lunau, K., Ren, Z.-X., Fan, X.-Q., Trunschke, J., Pyke, G.H. \& WANG, H. 2020: Nectar mimicry: a new phenomenon. - Scientific Reports 10: 7039.

Lunau, K. \& Verhoeven, C. 2017: Wie Bienen Blumen sehen - Falschfarbenaufnahmen von Blüten. - BiuZ 47: 120-127.

Martínez-Harms, J., Hadar, R., Márquez, N., Menzel, R., Shmida, A., Stavenga, D. G. \& Vorobyev, M. 2020: Enhanced UV-reflection facilitated a shift in the pollination system of the Red Poppy, Papaver rhoeas (Papaveraceae). Plants 9: 927.

Moyroud, E. \& Glover, B.J. 2016: The physics of pollinator attraction. New Phytol. 216: 350-354.

Moyroud, E., Wenzel, T., Middleton, R., Rudall, P. J., Banks, H., Reed, A., Mellers, G., Killoran, P., Westwood, M.M., Steiner, U., Vignolini, S. \& Glover, B.J. 2017: Disorder in convergent floral nanostructures enhances signalling to bees. - Nature 550: 469-474.

Ödeen, A. \& Håstad, O. 2010: Pollinating birds differ in spectral sensitivity. - J. Comp. Physiol. A 196: 91-96.

Osche, G. 1979: Zur Evolution optischer Signale bei Blütenpflanzen. - BiuZ 9: 161-170.

Papiorek, S., Junker, R.R., Lunau, K. 2014. Gloss, colour and grip: Multifunctional epidermal cell shapes in bee- and bird-pollinated flowers. - PLoS ONE 9; e112013.

Papiorek S., Junker, R.R., Alves-dos-Santos, I., Melo, G.A.R., Amaral-Neto, L.P., Sazima, M., Wolowski, M., Freitas, L. \& Lunau, K. 2016: Bees, birds and yellow flowers: Pollinator-dependent convergent evolution of UV-patterns. Plant Biology 18: 46-55.

Pohl, M., Watolla, T. \& Lunau, K. 2008: Anther-mimicking floral guides exploit a conflict between innate preference and learning in bumblebees (Bombus terrestris). - Behav. Ecol. Sociobiol. 63: 295-302.

Thüs, P., Lunau, K. \& Wester, P. 2020: Colour vision in sengis (Macroscelidea, Afrotheria, Mammalia): choice experiments indicate dichromatism. - Behaviour 157: 1127-1151.

Winter, Y., López, J. \& von Helversen, O. 2003: Ultraviolet vision in a bat. - Nature 425: 612-614.

\section{Anschrift des Autors}

Dr. Klaus Lunau, Dresdener Str. 11, 40822 Mettmann, E-Mail: Klaus.Lunau@hhu.de https://orcid.org/0000-0001-5184-4201 